\title{
Cyclotron resonance in antidot arrays
}

\author{
D. G. Polyakov, ${ }^{1, *}$ F. Evers, ${ }^{1}$ and I. V. Gornyi ${ }^{1,2, *}$ \\ ${ }^{1}$ Institut für Nanotechnologie, Forschungszentrum Karlsruhe, 76021 Karlsruhe, Germany \\ ${ }^{2}$ Institut für Theorie der Kondensierten Materie, Universität Karlsruhe, 76128 Karlsruhe, Germany
}

(Received 15 August 2001; published 13 March 2002)

\begin{abstract}
We study the dynamical properties of an electron gas scattered by impenetrable antidots in the presence of a strong magnetic field. We find that the line shape of the cyclotron resonance is very different from the Lorentzian and is not characterized by the Drude scattering rate. We show that the dissipative dynamical response of skipping orbits, $S_{c}(\omega)$, is broadened on a scale of the cyclotron frequency $\omega_{c}$ and has a sharp dip $\propto\left|\omega-\omega_{c}\right|$. For small antidots, $S_{c}(\omega)$ is strongly modulated with a period equal to $\omega_{c}$ and has sharp square-root singularities for a series of resonant frequencies. For large antidots, $S_{c}(\omega)$ has a hard gap at $\omega<\omega_{c}$ between two sharp peaks, associated, respectively, with edge states and free cyclotron orbits.
\end{abstract}

DOI: 10.1103/PhysRevB.65.125326

PACS number(s): 73.21.-b, 73.43.-f

\section{INTRODUCTION}

Progress in controlled fabrication of semiconductor nanostructures ${ }^{1}$ has revived interest in quasiclassical features of transport in a two-dimensional electron gas (2DEG). Since the Fermi wavelength of electrons in high-mobility heterostructures is usually small compared to the characteristic spatial scale of inhomogeneities, the transport properties of the 2DEG retain signatures of the underlying quasiclassical dynamics of the particles. In particular, transport in ballistic mesoscopic systems, where electrons are scattered specularly on the boundary of the system, has been investigated in terms of quasiclassical dynamics in considerable detail. ${ }^{2}$ In antidot (AD) arrays, potential barriers around the AD's can also be viewed as hard disks of size $\sim 10-10^{2} \mathrm{~nm}$ that reflect electrons specularly. If $\mathrm{AD}$ arrays are periodic (for a review see Refs. 3 and 4), the quasiclassical character of electron dynamics manifests itself in pronounced geometrical resonances, which are associated with the periodicity and lead, in particular, to commensurability peaks in the magnetoresistance. On the other hand, random AD arrays (for experimental work on dc transport see, e.g., Refs. 5-8) represent a remarkable disordered system in which the statistics of fluctuations of the random potential is strongly non-Gaussian (in contrast to the familiar case of smooth disorder in highmobility heterostructures, where the random potential at a given point is a sum of contributions from many impurities).

On the theoretical side, the recent interest in the quasiclassical dynamics of a 2DEG is to a large extent inspired by a variety of "non-Boltzmann" quasiclassical transport phenomena that occur in disordered systems with large-scale inhomogeneities. The term "non-Boltzmann" means that these phenomena, while being essentially classical, cannot be described by the Boltzmann kinetic equation (i.e., the collision-integral approximation is insufficient). They are due to correlations of scattering acts at the points where quasiclassical paths self-intersect, which gives rise to memory effects, not captured by Boltzmann transport theory. Most noticeably, the non-Markovian kinetics yields a wealth of anomalous magnetotransport phenomena in low magnetic fields, ${ }^{9-11}$ induces adiabatic localization of electrons in strong fields, ${ }^{12,13}$ and leads to a peculiar behavior of the magnetoresistivity in the Lorentz model of hard-disk scatterers. ${ }^{14-16}$ It is important that the quasiclassical nonBoltzmann corrections dominate over the quantum ones in systems with long-range disorder.

The non-Markovian character of kinetics that leads to the anomalous dc transport manifests itself also in the cyclotron resonance (CR). In particular, the adiabatic localization ${ }^{12,13}$ is predicted ${ }^{17}$ to yield a peculiar shape of the CR line: a narrow peak related to chaotic dynamics of delocalized electrons on top of an inhomogeneously broadened background coming from adiabatically localized electrons. The latter contribution dominates for large $B$ and gives the linewidth which depends nonmonotonically on $B$. This intricate picture should be contrasted with the case of white-noise disorder, where the linewidth is given by the scattering rate. ${ }^{18}$

In this paper, we consider the $\mathrm{CR}$ in $\mathrm{AD}$ arrays. We demonstrate that the CR line shape is very much different from the Lorentzian suggested by the Drude theory. The peculiarity of the dynamical response of the AD system is related to two factors which become important with increasing $B$ : the formation of "skipping orbits" of electrons bound to AD's and suppression of scattering for other electrons that do not participate in the process of skipping. We show that the CR is not characterized simply by the Drude scattering rate. Specifically, cyclotron orbits not colliding with AD's yield an infinitely sharp CR line, whereas skipping orbits give a contribution broadened on a scale of the cyclotron frequency $\omega_{c}$. The skipping-orbit contribution exhibits a remarkably rich behavior as a function of frequency $\omega$ : it has a sharp dip $\propto\left|\omega-\omega_{c}\right|$ at $\omega=\omega_{c}$ and, in the case of small AD's, is strongly modulated with a period equal to $\omega_{c}$. The modulation yields exact zeros of the CR response in a dilute $\mathrm{AD}$ array. In addition to the zeros, a series of sharp singularities is developed in the wings of the CR line. For large AD's, the dynamical response has a hard gap at $\omega<\omega_{c}$ between two sharp peaks, associated, respectively, with edge states and free cyclotron orbits.

The paper is organized as follows. In Sec. II, we derive an exact expression for the CR line shape in the limit of large $B$, which is then analyzed in two essentially different cases of small (Sec. III) and large (Sec. IV) AD's. In Sec. V, we consider moderately strong magnetic fields (moderately in the sense that skipping orbits bound to different AD's can 
overlap). We add remarks bearing on experiments and the role of electron-electron interactions in Sec. VI. Throughout the paper, the analytical calculation is complemented by the results of numerical simulations.

\section{LARGE-B LIMIT: INSULATING PHASE}

We start by considering the limit of large $B$, namely, $n_{S} R_{c}^{2} \ll 1$, where $n_{S}$ is the concentration of AD's and $R_{c}$ the cyclotron radius. We model AD's by hard disks and assume that the array of AD's is dilute, i.e., $n_{S} a^{2} \ll 1$, where $a$ is the radius of the disks. In this model (known as the Lorentz gas), if $R_{c}$ is smaller than a critical value of the order of $n_{S}^{-1 / 2}$, all quasiclassical trajectories get localized and the dissipative dc conductivity $\sigma_{x x}(\omega=0)$ vanishes at zero temperature exactly. ${ }^{14,15}$ The localization is developed through the formation of disconnected clusters of trajectories that do not extend beyond a finite area. In random $\mathrm{AD}$ arrays, the metalinsulator transition is "second order," so that as one goes deeper into the insulating phase with increasing $B$, the size of the critical clusters decreases continuously. Eventually, far away from the critical point, for $n_{S} R_{c}^{2} \ll 1$, trajectories that collide with two or more AD's become very rare. In this limit, most electrons do not collide with AD's whatsoever and give a $\delta$-function CR line at $\omega=\omega_{c}$. Most of those that collide move in skipping orbits around a single AD. It follows that for large $B$ the dynamical response at $\omega \neq \omega_{c}$ is determined by the skipping orbits. Clearly, this conclusion is true both for random and periodic $\mathrm{AD}$ arrays.

Let us calculate the dynamical response associated with skipping orbits. Since in the phase space of the Lorentz gas there is a well-defined separatrix between free cyclotron orbits and trajectories colliding with AD's, we write the dissipative conductivity as a sum of two terms,

$$
\operatorname{Re} \sigma_{x x}(\omega)=\frac{1}{2} e^{2} \rho_{0}\left[p D_{f}(\omega)+(1-p) D_{c}(\omega)\right],
$$

where

$$
p=\exp \left(-2 \pi / \omega_{c} \tau_{0}\right)
$$

is the probability to close the cyclotron orbit without suffering a collision, $\tau_{0}=1 / 2 v_{F} n_{S} a$ the collision time, and $\rho_{0}$ the density of states for free electrons at $B=0$. The symmetrized functions $\quad D_{f, c}(\omega)=v_{F}^{2}\left[S_{f, c}(\omega)+S_{f, c}(-\omega)\right] / 2$ are the velocity-velocity correlators for free electrons and electrons colliding with AD's, respectively; $v_{F}$ is the Fermi velocity. In the above, we have assumed that many Landau levels are occupied, so that $k_{F} R_{c} \gg 1$, where $k_{F}$ is the Fermi wave vector, and that AD's are large enough, in the sense that $k_{F} a$ $\gg 1$, which means that AD's scatter electrons specularly. It is also important to us that under these conditions the density of states of electrons scattered by AD's is not affected by the Landau quantization and is given simply by $(1-p) \rho_{0}$.

The dynamical response to a circularly polarized (CRactive) perturbation is given by $S_{f, c}(\omega)$. For free electrons we have $S_{f}(\omega)=\pi \delta\left(\omega-\omega_{c}\right)$, while for electrons skipping around an $\mathrm{AD}$

$$
\begin{aligned}
S_{c}(\omega)= & \int_{0}^{\infty} d t\left\langle\operatorname { c o s } \left[\left(\omega_{c}-\omega\right) t\right.\right. \\
& \left.\left.+2 \theta \sum_{m=1}^{\infty} \Theta(t+\Delta t-m T)\right]\right\rangle_{\Delta t, r},
\end{aligned}
$$

where $\Theta(t)$ is the step function and

$$
\langle\rangle_{\Delta t}=\frac{\omega_{c}}{2 \pi} \int_{0}^{T(r)} d \Delta t, \quad\langle\rangle_{r}=\frac{1}{2 R_{c} a} \int_{\left|R_{c}-a\right|}^{R_{c}+a} r d r
$$

denotes averaging over the initial phase $\omega_{c} \Delta t$ and the distance $r$ between the centers of the AD and the cyclotron orbit. The normalization of the integral \langle\rangle$_{\Delta t}$ takes care of the "exclusion volume" free of electrons because of the presence of AD's. The angle of incidence $\theta(r)$ (the angle between the trajectory and the tangent to the surface of the AD at the collision point) and the time $T(r)$ between two successive collisions read

$$
\begin{gathered}
\cos \theta(r)=\frac{r^{2}-R_{c}^{2}-a^{2}}{2 R_{c} a}, \\
\cos \frac{\omega_{c} T(r)}{2}=\frac{a^{2}-R_{c}^{2}-r^{2}}{2 R_{c} r} .
\end{gathered}
$$

Since $r$ is an integral of motion for orbits skipping around a disk, $\theta(r)$ and $T(r)$ are the same for each collision.

Doing the integrations over $t$ and $\Delta t$ and summing over $m$ in Eq. (3), we get an identically zero response at the cyclotron frequency for colliding electrons, $S_{c}\left(\omega_{c}\right) \equiv 0$, and

$$
S_{c}(\omega)=\frac{\omega_{c}}{\left(\omega_{c}-\omega\right)^{2}}\left\langle\sin ^{2} \theta(r) \sum_{n=-\infty}^{\infty} \delta\left[f_{\omega}(r)+\pi n\right]\right\rangle_{r}
$$

$$
=\frac{\omega_{c}}{2 R_{c} a\left(\omega_{c}-\omega\right)^{2}} \sum_{n} \frac{r_{n} \sin ^{2} \theta\left(r_{n}\right)}{\left|f_{\omega}^{\prime}\left(r_{n}\right)\right|}
$$

for $\omega \neq \omega_{c}$. Here $f_{\omega}(r)=\frac{1}{2}\left(\omega_{c}-\omega\right) T(r)+\theta(r)$ and $r_{n}(\omega)$ are roots of the equation $f_{\omega}(r)+\pi n=0$. The summation in Eq. (8) runs over $r_{n}$ that satisfy $\left|R_{c}-a\right|<r_{n}<R_{c}+a$.

Equation (7) tells us that the dynamical response is due to resonant orbits. There is no broadening of the contribution of each of the orbits and, accordingly, the shape of the CR line is given by the density of states of the resonant orbits. The meaning of the resonance condition $\omega=\omega_{c}+2(\theta+\pi n) T^{-1}$ is that the change of the total phase (cyclotron phase + phase of the ac field + scattering phase) should be a multiple of $2 \pi$ between two collisions. It is worth noting that, contrary to what one might expect, the resonant orbits are not periodic two-dimensional orbits. The formation of periodic orbits is indeed important for the understanding ${ }^{19,20}$ of transport through periodic arrays at weak $B$, where geometric resonances in the dc magnetoresistance ${ }^{19-21}$ and in the position of the main CR peak ${ }^{22}$ in the photoconductivity are observed. However, two-dimensional periodic orbits do not play any particular role in Eqs. (7) and (8) (so that their separate treat- 
ment is not justified in the calculation of the response of skipping orbits bound to a single AD). This conclusion should be contrasted with the theoretical approach of Ref. 22 , where it has been suggested that the skipping-orbit response is dominated by peaks at $\omega$ corresponding to periodic orbits.

To analyze $S_{c}(\omega)$, it is convenient to rewrite Eq. (8) and the resonance condition in terms of the function $g_{\omega}(\theta)$ $=f_{\omega}[r(\theta)]$ defined on the interval $0<\theta<\pi$ :

$$
\begin{gathered}
S_{c}(\omega)=\frac{\omega_{c}}{2\left(\omega_{c}-\omega\right)^{2}} \sum_{n} \frac{\sin ^{3} \theta_{n}}{\left|g_{\omega}^{\prime}\left(\theta_{n}\right)\right|}, \\
g_{\omega}(\theta)=\frac{1}{2}\left(\omega_{c}-\omega\right) T(\theta)+\theta, \\
g_{\omega}\left(\theta_{n}\right)+\pi n=0 .
\end{gathered}
$$

The time between collisions $T(\theta)$ behaves in an essentially different way depending on whether the ratio $R_{c} / a$ is larger or smaller than unity.

\section{SMALL ANTIDOTS}

Let us first study the case $R_{c} \gg a$ by expanding $T(\theta)$ in powers of $a / R_{c}$. Combining Eqs. (5) and (6) we get

$$
T(\theta)=\frac{2 \pi}{\omega_{c}}-\frac{2 a}{v_{F}}\left(\sin \theta-\frac{a}{2 R_{c}} \sin 2 \theta+\cdots\right),
$$

with $T(0)=T(\pi) \equiv 2 \pi / \omega_{c}$. To find the roots $\theta_{n}$ at $\mid \omega$ $-\omega_{c} \mid \ll v_{F} / a$, one can retain only the first (unperturbed) term in Eq. (12), which yields, for a given $\omega$, a single solution $\theta_{n} \simeq \pi\left(\omega / \omega_{c}-\left[\omega / \omega_{c}\right]\right)$ and $g_{\omega}^{\prime}\left(\theta_{n}\right) \simeq 1$. Here $\left[\omega / \omega_{c}\right]$ is the integer part of $\omega / \omega_{c}$. Substituting these expressions in Eq. (9) we find

$$
\begin{gathered}
S_{c}(\omega)=\frac{\omega_{c}}{2\left(\omega_{c}-\omega\right)^{2}} \sin ^{3} \frac{\pi\left(\omega-n \omega_{c}\right)}{\omega_{c}}, \\
D_{c}(\omega)=v_{F}^{2} \frac{\omega_{c}^{2}+\omega^{2}}{\left(\omega_{c}+\omega\right)^{2}} S_{c}(\omega)
\end{gathered}
$$

for $n \omega_{c}<\omega<(n+1) \omega_{c}$ and $|n| \ll R_{c} / a$. We thus see that the CR line exhibits a strong modulation; namely, $S_{c}(\omega)$ has zeros at $\omega=n \omega_{c} .{ }^{23}$ Note that the zeros are exact even if higher-order terms in powers of $a / R_{c}$ are taken into account. These zeros correspond to resonant orbits with $\theta \rightarrow 0$ and $\theta$ $\rightarrow \pi$, which go along the tangent at the point where they touch the AD. In the vicinity of the zeros $S_{c}(\omega)$ behaves as $\left|\omega-n \omega_{c}\right|^{3}$ for any $n \neq 1$, including the dc limit $n=0$. At the point $\omega=\omega_{c}, S_{c}(\omega)$ vanishes as $\left|\omega-\omega_{c}\right|$. The envelope of the oscillations (13) falls off with increasing $\omega$ as ( $\omega$ $\left.-\omega_{c}\right)^{-2}$, similarly to the conventional Lorentzian.

The total response of the 2DEG scattered by AD's of small radius $a\left(R_{c} / a \gg 1\right.$ and $\left.|\omega| \ll v_{F} / a\right)$ is thus a sum of two parts: a sharp peak associated with $S_{f}(\omega)$ and a series of broader peaks given by $S_{c}(\omega)$, whose width is $\sim \omega_{c}$. The oscillatory behavior of $D_{c}(\omega)$ is shown in Fig. 1. One can

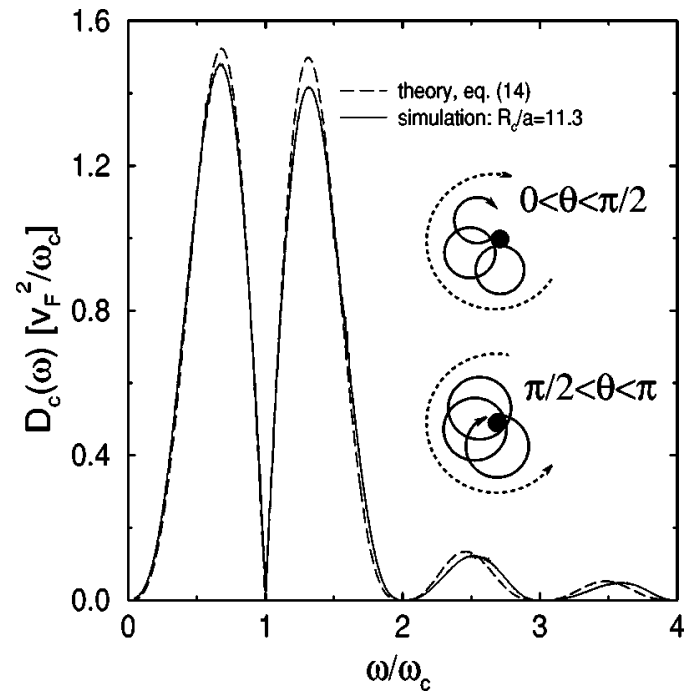

FIG. 1. Dynamical response of skipping orbits for $R_{c} / a \gg 1$ and $\omega \ll v_{F} / a$ exhibits oscillatory behavior with a characteristic double peak around zero at $\omega=\omega_{c}$. Dashed line: $D_{c}(\omega)$ in units of $v_{F}^{2} / \omega_{c}$ according to Eqs. (13) and (14). Solid line: numerical simulation for $R_{c} / a \simeq 11.3$. The insets illustrate the different sense of rotation for resonant skipping orbits with $1<\omega / \omega_{c}<3 / 2$ (upper panel) and $1 / 2$ $<\omega / \omega_{c}<1$ (lower panel).

see that the first two peaks of $D_{c}(\omega)$ that occur between $\omega$ $=0$ and $\omega=2 \omega_{c}$ are much higher than those for larger $\omega$. As a result, the response of skipping orbits with $R_{c} / a \gg 1$ looks like a double peak split up at $\omega=\omega_{c}$. Note that the resonant value of $\theta$ tends to $\pi$ if one approaches $\omega_{c}$ from the left $\left(\omega \rightarrow \omega_{c}-0\right)$, whereas it tends to 0 if one does so from the right $\left(\omega \rightarrow \omega_{c}+0\right)$. Accordingly, the two parts of the double peak for $1<\omega / \omega_{c}<3 / 2$ (where the resonant angles $0<\theta$ $<\pi / 2$ ) and $1 / 2<\omega / \omega_{c}<1$ (where $\pi / 2<\theta<\pi$ ) differ in the direction in which the guiding centers of the resonant skipping orbits rotate around the AD (see the insets in Fig. 1).

The wing of the principal double peak (Fig. 1) exhibits nontrivial behavior. As $\omega$ increases, $S_{c}(\omega)$ in intervals between two adjacent zeros gets more and more asymmetric with maxima shifting towards the higher- $|\omega|$ boundary of the intervals. Eventually, when $|\omega|$ reaches the critical frequency $v_{F} / a$, the behavior of $S_{c}(\omega)$ acquires qualitatively new features. At $|\omega|>v_{F} / a, g_{\omega}(\theta)$ as a function of $\theta$ becomes nonmonotonic, which can be seen from Eqs. (10) and (12). This leads to the appearance of multiple roots $\theta_{n}$ for a given $\omega$ (in contrast to a single root at $\left.|\omega|<v_{F} / a\right)$. Because of the multiple roots, the zeros in $S_{c}(\omega)$ disappear for $|\omega|>v_{F} / a$, since the condition $\theta_{n}=0$ or $\pi$ now cannot be met for all roots simultaneously (so that there is a finite number of zeros, namely, $2 R_{c} / a$ zeros at $R_{c} \gg a$ ). Moreover, the nonmonotonic behavior of $g_{\omega}(\theta)$ yields singularities in the line shape associated with resonant orbits for which the derivative $g_{\omega}^{\prime}\left(\theta_{n}\right)$ vanishes in the denominator of Eq. (9).

Let us analyze the high- $\omega$ limit $|\omega| \gg v_{F} / a$. In this case, one can neglect the last term on the right-hand side of Eq. (10) and represent the equation for $\theta_{n}$ at $n \gg 1$ in the form $\left(\omega a / v_{F}\right) \sin \theta_{n}+\pi\left(n-\omega / \omega_{c}\right)=0$ with $g_{\omega}^{\prime}\left(\theta_{n}\right)$ $\simeq\left(\omega a / v_{F}\right) \cos \theta_{n}$. One sees that now for a given $\omega$ there are 


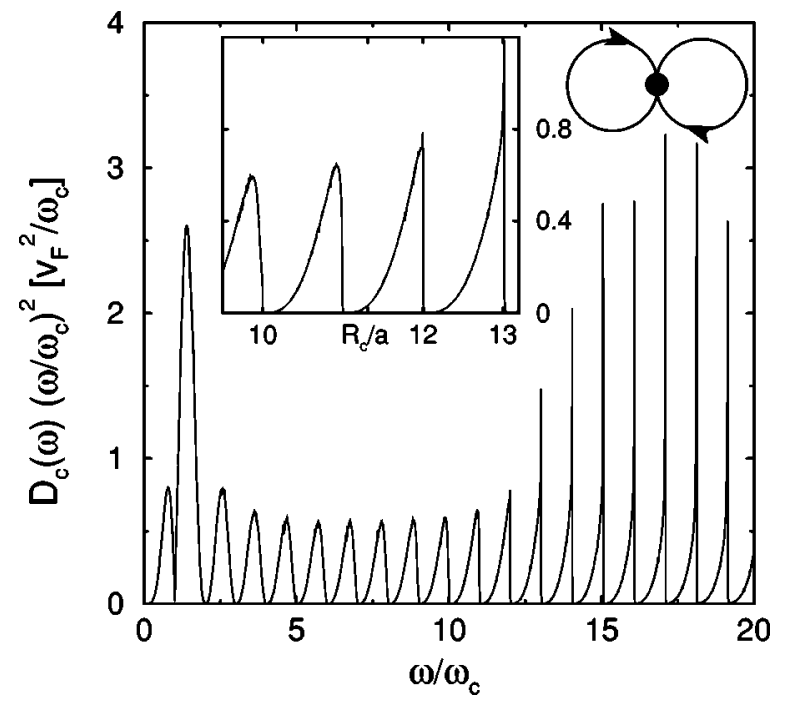

FIG. 2. Dynamical response of skipping orbits with $R_{c} / a \gg 1$ in the tail of the principal double peak (shown in Fig. 1). The oscillating curve represents the product $D_{c}(\omega) \times\left(\omega / \omega_{c}\right)^{2}$ in units of $v_{F}^{2} / \omega_{c}$ as obtained from the numerical simulation for $R_{c} / a \simeq 11.3$. The inset magnifies the region near $\omega / \omega_{c}=R_{c} / a$, where zeros of $D_{c}(\omega)$ disappear and simultaneously a series of square-root singularities starts [Eqs. (15) and (16)]. It also shows the resonant orbit corresponding to the singular frequency.

$2|\omega| a / \pi v_{F} \gg 1$ roots $\theta_{n}$. Transforming to the continuous limit in the summation over $\theta_{n}$ [Eq. (9)] we obtain a regular part of $S_{c}(\omega)$ for $|\omega| \gg v_{F} / a$ :

$$
S_{c}^{r e g}(\omega)=\frac{2}{3 \pi} \frac{\omega_{c}}{\omega^{2}}
$$

The most prominent feature on top of the smoothly varying background (15) is the appearance at $|\omega|>v_{F} / a$ of sharp singularities ("spikes") in $S_{c}(\omega)$. The spikes occur every time $g_{\omega}^{\prime}(\theta)$ vanishes for one of the roots $\theta_{n}$. Note that the singularity frequencies coincide for $S_{c}(\omega)$ and $S_{c}(-\omega)$. At $|\omega| \gg v_{F} / a, g_{\omega}(\theta)$ reaches maximum at $\theta=\theta_{\max } \simeq \pi / 2$ $+a / R_{c}+v_{F} / \omega a$, close to $\pi / 2$. It follows that the skipping orbits that yield the spikes hit the surface of an $\mathrm{AD}$ at almost a right angle, so that the center of the orbits flicks from one side of the $\mathrm{AD}$ to the other after each collision. Expanding around the maximum and substituting two (almost degenerate) roots $\theta_{n}(\omega)$ of Eq. (11) which are close to $\theta_{\max }$, we find $S_{c}(\omega)$ in the vicinity of the singularities for $|\omega| \gg v_{F} / a$ :

$$
S_{c}^{\operatorname{sing}}(\omega)=\frac{\omega_{c}}{\omega^{2}}\left(\frac{v_{F}}{2 \pi a|\omega|}\right)^{1 / 2}\left|\frac{\omega_{c}}{\omega_{n}-\omega}\right|^{1 / 2} \Theta\left[\left(\omega_{n}-\omega\right) \operatorname{sgn} \omega\right] .
$$

The frequencies $\omega_{n}$ for $|\omega| \gg v_{F} / a$ and $R_{c} \gg a$ are given by $\omega_{n} \simeq \omega_{c}(n+3 / 2)\left(1+a / \pi R_{c}\right)$. Note that the period of the sequence of spikes is larger than $\omega_{c}$ (but close to $\omega_{c}$ at $R_{c}$ $\gg a)$. The behavior of $S_{c}(\omega)$ for large $\omega a / v_{F}$ is illustrated in Fig. 2. The square-root singularities at $\omega \rightarrow \omega_{n}$ that appear for $\omega>v_{F} / a$ (i.e., for $\omega / \omega_{c}>R_{c} / a$ ) are clearly seen. The region of $\omega \simeq v_{F} / a$ is blown up in the inset to show the sudden start of the series of singularities when $\omega$ becomes larger than $v_{F} / a$.

\section{LARGE ANTIDOTS}

We now turn to the case $R_{c} \ll a$. As we will see, $S_{c}(\omega)$ shows completely different behavior in the two limits of large and small $R_{c} / a$. The function $T(\theta)$ expanded in powers of $R_{c} / a$ reads

$$
T(\theta)=\frac{2(\pi-\theta)}{\omega_{c}}+\frac{2 R_{c}}{\omega_{c} a}\left(\sin \theta-\frac{3 R_{c}}{4 a} \sin 2 \theta+\cdots\right),
$$

with $T(0) \equiv 2 \pi / \omega_{c}$ and $T(\pi) \equiv 0$. Clearly, if one sends $R_{c} / a \rightarrow 0, S_{c}(\omega)$ describes the dynamical response of trajectories skipping along a straight line ("edge states"). Using Eq. (17) in this limit, we get $1+\left[|\omega| / \omega_{c}\right]$ roots $\theta_{n}=\pi-(n$ +1) $\pi \omega_{c} /|\omega|$ with $0 \leqslant n+1 \leqslant\left[|\omega| / \omega_{c}\right]$, for which $g_{\omega}^{\prime}\left(\theta_{n}\right)$ $=\omega / \omega_{c}$. Substitution in Eq. (9) yields

$$
S_{c}(\omega)=\frac{\omega_{c}^{2}}{2|\omega|\left(\omega_{c}-\omega\right)^{2}} \sum_{n=0}^{\left[|\omega| / \omega_{c}\right]-1} \sin ^{3} \frac{(n+1) \pi \omega_{c}}{|\omega|}
$$

and $D_{c}(\omega)$ which is related to $S_{c}(\omega)$ by Eq. (14). Since the limits $\omega \rightarrow 0$ and $R_{c} / a \rightarrow 0$ do not commute with each other, one should be careful about the behavior of $S_{c}(\omega)$ at $\omega \rightarrow 0$. In fact, $S_{c}(\omega)$ for the edge states has a $\delta(\omega)$ term in the dc limit, so that Eq. (18) correctly describes the response of a large AD with $R_{c} / a \rightarrow 0$ only at $\omega \neq 0$ (see below).

As follows from Eq. (18), in a striking difference with the case of large $R_{c} / a$, there appears a gap with $S_{c}(\omega)=0$ for $0<|\omega|<\omega_{c}$. No dissipation occurs with increasing $\omega$ until $|\omega|$ exceeds $\omega_{c}$. At the edge of the gap, $S_{c}(\omega)$ vanishes linearly as $|\omega|-\omega_{c}$. Also, in contrast to the case of large $R_{c} / a, S_{c}(\omega)$ given by Eq. (18) has neither zeros [cf. Eq. (13)] nor singularities [cf. Eq. (16)] for $|\omega|>\omega_{c}$. In the limit of large $|\omega| \gg \omega_{c}$, replacing the summation in Eq. (18) by integration leads to $S_{c}(\omega) \propto \omega^{-2}$ which behaves according to Eq. (15).

As mentioned above, in addition to Eq. (18) there is a $\delta(\omega)$ peak in $S_{c}(\omega)$ for edge states with $R_{c} / a \rightarrow 0$. To calculate the dynamical response of AD's of a large but finite radius, one should take into account terms of higher order in $R_{c} / a \ll 1$ in the expansion (17), which leads to a broadening of the peak in a finite range of frequency, namely, $0<\omega$ $<v_{F} / a$. This broadening is governed by the root $\theta_{-1}$ which obeys the equation

$$
\frac{\omega a}{v_{F}}=\frac{\sin \theta_{-1}}{\pi-\theta_{-1}}
$$

with $g_{\omega}^{\prime}\left(\theta_{-1}\right)=\omega_{c}^{-1}\left(\omega-v_{F} \cos \theta_{-1} / a\right)$, where we retained only the term linear in $R_{c} / a$ in Eq. (17). We see that a solution of Eq. (19) exists only in the above-mentioned interval of $\omega$. Within this interval $S_{c}(\omega)$ reads 


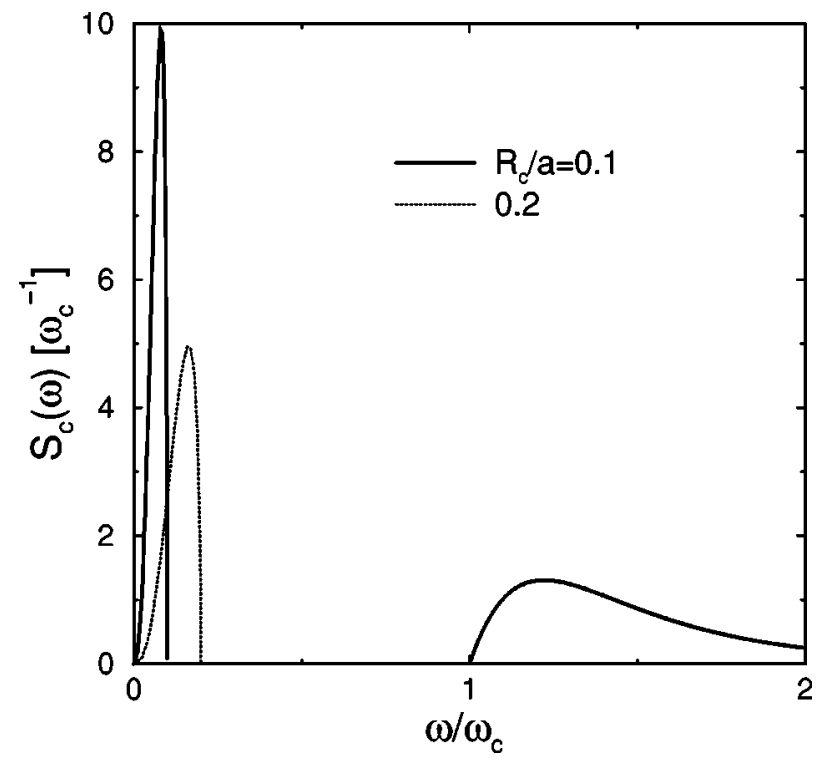

FIG. 3. Dynamical response of skipping orbits with $R_{c} / a \ll 1$ (edge states). The sharp low-frequency peak is separated from the broad peak at $\omega \sim \omega_{c}$ by a hard gap. The curve for $\omega>\omega_{c}$ is $S_{c}(\omega)$ in units of $\omega_{c}^{-1}$ calculated according to Eq. (18). The peak at 0 $<\omega<v_{F} / a$ is a solution of Eqs. (19) and (20) for $R_{c} / a=0.1$ (solid line) and $R_{c} / a=0.2$ (dotted line).

$$
S_{c}(\omega)=\frac{a}{2 v_{F}} \frac{\sin ^{3} \theta_{-1}}{\cos \theta_{-1}+\omega a / v_{F}} .
$$

Combining Eqs. (19) and (20) we obtain the asymptotic behavior of $S_{c}(\omega)$ near the ends of the interval: $S_{c}(\omega)$ vanishes as $\omega^{3}$ at $\omega \rightarrow 0$ and as $\left(v_{F} / a-\omega\right)^{1 / 2}$ at $\omega \rightarrow v_{F} / a$. The total weight of the peak at small $\omega$ is given by $\int_{0}^{v_{F} / a} d \omega S_{c}(\omega)$ $=\int_{0}^{\pi} d \theta(2 \theta)^{-1} \sin ^{3} \theta \simeq 0.485$.

The dynamical response of the $2 \mathrm{DEG}$ in the case of relatively large AD's with $R_{c} / a \ll 1$ is thus a sum of three peaks: two sharp peaks, one centered at low frequency $\omega \sim v_{F} / a$, the other at higher frequency $\omega=\omega_{c}$ (the latter is related to free cyclotron orbits), plus a broad peak of width $\sim \omega_{c}$ whose edge coincides with $\omega_{c}$. The behavior of the contribution of skipping orbits, $S_{c}(\omega)$, is illustrated in Fig. 3 .

Note that the hard gap in $S_{c}(\omega)$ gets narrower but remains exact at small $R_{c} / a$. In fact, the hard gap survives with increasing $R / a$ up until $R_{c}$ becomes equal to $a$, at which point the geometry of skipping changes in a qualitative way. Specifically, at $R_{c}<a$ skipping orbits propagate in only one direction around the $\mathrm{AD}$, whereas for $R_{c}>a$ they propagate in both. As a result, the hard gap at $|\omega|<\omega_{c}$ disappears (transforms into a soft gap $\left.\propto\left|\omega-\omega_{c}\right|\right)$ for $R_{c}>a$ and simultaneously infinitely many singularities pop up at $|\omega|>\omega_{c}$. With further increasing $R_{c} / a$, zeros in $S_{c}(\omega)$ proliferate in the finite range of frequency $|\omega|<v_{F} / a$, as explained above.

\section{MODERATELY STRONG $B$ : METALLIC PHASE}

The above analysis of the dynamical response of a single AD applies directly to the case of a strong magnetic field, $n_{S} R_{c}^{2} \ll 1$. In this limit, a skipping orbit is bound to a single
$\mathrm{AD}$ for an infinitely long time. With decreasing $B$, the scattering processes that involve collisions of a skipping orbit with many AD's become essential and at a critical value of the parameter $n_{S} R_{c}^{2} \sim 1$ a metal-insulator transition occurs. On the conducting side of the transition, the skipping orbits become delocalized by hopping from one AD to another. Deep in the metallic phase, for $n_{S} R_{c}^{2} \gg 1$, the characteristic hopping rate is $\tau_{0}^{-1}$ and the dc conductivity $\sigma_{x x}(0)$ is given by the second term in Eq. (1) with ${ }^{15} D_{c}(0)=v_{F}^{2} \tau(x) /[1$ $\left.+\omega_{c}^{2} \tau^{2}(x)\right]$ parametrized by $x=\omega_{c} \tau_{0}$, where $\tau(\infty)=\tau_{0}$ and $\tau(0)=3 \tau_{0} / 4$. At zero $B$, Boltzmann theory works perfectly well in the hydrodynamic limit $\left(n_{S} \rightarrow \infty, n_{S} a=\right.$ const $)$, but due to the factor of $1-p$ in Eq. (1), $\sigma_{x x}(0)$ falls off as $B^{-3}$ in the conducting phase, one power of $B$ faster than in Boltzmann theory. One might think that, apart from this factor, the dynamical response at finite $B$ will also be similar to that in the Boltzmann approach. The latter is simply the zero- $B$ Lorentzian with a shifted frequency $\omega \rightarrow \omega-\omega_{c}$. In fact, however, the behavior of $S_{c}(\omega)$ in the conducting phase is completely different from the Lorentzian; see below.

While $S_{c}(\omega)$ in the de limit shows the metal-insulator transition, at larger $\omega$ the function $S_{c}(\omega)$ in the conducting phase retains, provided $\omega_{c} \tau_{0} \gg 1$, the main features of the single-AD response with $R_{c} / a \gg 1$ [Eqs. (12)-(16)]. The oscillatory behavior of the line shape with sharp dips at $\omega$ $=n \omega_{c}$ remains almost unchanged at $\omega_{c} \tau_{0} \gg 1$, since in this limit skipping orbits experience many collisions with a single AD before they "change" to another one. Clearly, in contrast to Eq. (13), $S_{c}(\omega)$ has no exact zeros any more; however, the behavior of $S_{c}(\omega)$ is modified only in a close vicinity of the points $\omega=n \omega_{c}$. In particular, because of the broadening of the resonances due to the hopping between AD's, the linear vanishing of $S_{c}(\omega) \propto\left|\omega-\omega_{c}\right|$ near $\omega=\omega_{c}$ is cut off at $\left|\omega-\omega_{c}\right| \sim \tau_{0}^{-1}$. On the other hand, the cubic vanishing of $S_{c}(\omega) \sim|\omega|^{3} / \omega_{c}^{4}$ matches $S_{c}(0) \sim\left(\omega_{c}^{2} \tau_{0}\right)^{-1}$ in the dc limit, which establishes the scale $|\omega| \sim \tau_{0}^{-1}\left(\omega_{c} \tau_{0}\right)^{2 / 3}$ on which the frequency dispersion of the conductivity becomes strong. A linear zero-frequency anomaly ${ }^{24} S_{c}(\omega)-S_{c}(0)$ $\propto|\omega|$ appears in the metallic phase. Notice that, because of the anomalously strong broadening of the CR line, the crossover to the regime $\sigma_{x x}(\omega) \gg \sigma_{x x}(0)$ occurs with increasing $\omega$ at much smaller (for $\omega_{c} \tau_{0} \gg 1$ ) frequency than in the Drude regime, where the characteristic scale is $\omega_{c}$.

The hopping between AD's also cuts off the square-root singularities (16) [they only survive for isolated AD's, whose contribution is suppressed by the factor of $\left.\exp \left(-4 \pi n_{S} R_{c}^{2}\right)\right]$. Note that the substitution $\omega \rightarrow \omega+i / \tau_{0}$ in Eq. (16) is only correct for $v_{F} / a \ll \omega \ll v_{F} R_{c} / a^{2}$. For larger $\omega$, the effective collision rate for the resonant orbits is renormalized, $\tau_{0}^{-1}$ $\rightarrow \widetilde{\tau}_{0}^{-1}$, since after each two collisions with a given AD the center of the resonant orbit is shifted by a distance $\delta R$ $\sim R_{c} v_{F} / a \omega$, which is much smaller than $a$ for the large frequencies. It follows that $\tilde{\tau}_{0}$ increases with $\omega, \tilde{\tau}_{0} / \tau_{0} \sim a / \delta R$, so that the singularities are cut off on the smaller scale of $\tilde{\tau}_{0}^{-1}$. Note that after the collision with another AD the center of the orbit is shifted by $\sim R_{c}(\delta R / a)^{1 / 2}$, which makes the orbit nonresonant. 
The smearing of the soft gaps around the zeros of $S_{c}(\omega)$ can be clearly seen in the hydrodynamic limit $n_{S} \rightarrow \infty, a$ $\rightarrow 0$ with $n_{S} a$ held fixed. In this limit, the kinetic problem allows for an exact solution, ${ }^{15,16}$ which we reproduce for convenience in the following form:

$$
S_{c}(\omega)=\frac{1}{\omega_{c}-\omega} \operatorname{Re}\left[\frac{1-p e^{-2 \pi i \omega / \omega_{c}}}{1-p} \frac{i \Sigma(\omega)}{\omega-\omega_{c}-\Sigma(\omega)}\right] \text {, }
$$

with

$$
\Sigma(\omega)=\frac{1}{\tau_{0}} \int_{0}^{\pi} d \theta \sin ^{2} \theta \frac{e^{i \theta}}{1-p e^{2 i g_{\omega}(\theta)}}
$$

Here $g_{\omega}(\theta)$ is given by Eq. (10) with $T(\theta)=2 \pi / \omega_{c}$, since in the hydrodynamic limit $R_{c} / a \rightarrow \infty$. Sending $\omega_{c} \tau_{0} \rightarrow \infty$ (i.e., $p \rightarrow 1$ ) generates a pole on the real axis of $\theta$ in the integrand of the self-energy (22), which yields $\Sigma(\omega)$ $=\left(\pi / 2 \tau_{0}\right) \sin ^{2} \theta e^{i \theta}$ with $\theta=\pi\left(\omega / \omega_{c}-\left[\omega / \omega_{c}\right]\right)$. The latter are precisely the $\theta$ 's defining the resonant orbits in Eq. (13). Using the above expression for $\Sigma(\omega)$ at $\omega_{c} \tau_{0} \rightarrow \infty$ in Eq. (21) indeed yields our Eq. (13), derived for the insulating phase. Clearly, no singularities [Eq. (16)] occur in the hydrodynamic limit. Note that the metal-insulator transition takes place in a dilute $\mathrm{AD}$ array at $R_{c} / a \gg 1$. Therefore, provided $\omega_{c} \tau_{0} \gg 1$ (which is the conventional condition for a developed CR resonance), $S_{c}(\omega)$ is in fact described by Eq. (13) not only in the metallic phase but also in the critical region of the metal-insulator transition, except for a very close vicinity of the zeros of $S_{c}(\omega)$.

In Fig. 4, we show results of our numerical simulation of ac transport in a very dilute $\mathrm{AD}$ array with $n_{S} a^{2} \simeq 0.62$ $\times 10^{-3}$, which corresponds to the mean free path $l_{S}$ $=3 / 8 n_{S} a \simeq 600 a$, tuned through the metal-insulator transition by changing magnetic field. The upper curve corresponds to the smallest $B$, with $l_{S} / R_{c} \simeq 2.12$. One sees that, contrary to what one would expect from the conventional Boltzmann theory, although the dc value $\sigma_{x x}(0)$ is only $\sim 6$ times smaller than that at zero $B$, a very narrow peak at $\omega$ $=\omega_{c}$ is developed, associated with free cyclotron orbits. As $B$ increases, this peak acquires more weight, while remaining very sharp. Next, in striking contrast to Boltzmann theory, the oscillations of $\sigma_{x x}(\omega)$, related to skipping orbits, become more and more pronounced with growing $B$. The doublepeak structure around the linear gap near $\omega=\omega_{c}$ is clearly seen, as are deep minima at $\omega$ equal to multiples of $\omega_{c}$. For large $l_{S} / R_{c}$, the numerically obtained behavior of $\sigma_{x x}(\omega)$ is in a good agreement with Eqs. (1), (13), and (14).

As follows from the comparison of Eqs. (13), (21), and (22), the dissipation at $\omega \neq \omega_{c}$ for finite $\omega_{c} \tau_{0}$ is due to resonant orbits (11) broadened by the hopping between different AD's. It is interesting to note that there appear two kinds of resonant behavior of the dynamical response in the metallic phase: first, the last factor in Eq. (21) resembles a resonance at $\omega \simeq \omega_{c}$ broadened by $\operatorname{Im} \Sigma$; on the other hand, the broadening itself is due to resonant orbits, for which the denominator in Eq. (22) is close to zero.

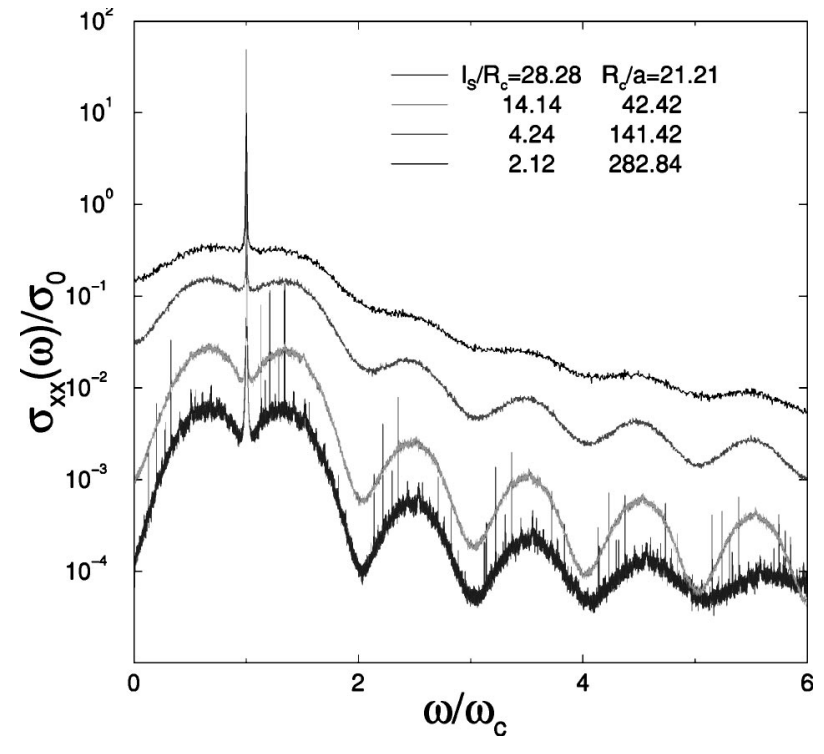

FIG. 4. Dynamical conductivity $\operatorname{Re} \sigma_{x x}(\omega)$ as a function of $\omega / \omega_{c}$ in units of the zero- $B$ zero- $\omega$ value $\sigma_{0}$ in a dilute antidot array as obtained by the numerical simulation. The transport mean free path at zero $B$ is $l_{S} \simeq 600 a$. Different curves correspond to different $B$. The ratio of $l_{S} / R_{c}$ changes from 2.12 for the upper curve to 4.24 , to 14.14 , up to 28.28 for the lower curve. Even for the relatively weak field with $l_{S} / R_{c}=2.12$ a very sharp peak at $\omega$ $=\omega_{c}$ is clearly seen. With increasing $B$, the broad double peak around $\omega=\omega_{c}$ gets more pronounced, as does the oscillatory behavior of $\sigma_{x x}(\omega)$ for higher $\omega$. For large $l_{S} / R_{c}=28.28$, the numerically obtained $\operatorname{Re} \sigma_{x x}(\omega)$ agrees well with Eqs. (1), (13), and (14).

\section{DISCUSSION}

In the above, we have calculated the quasiclassical dynamical conductivity of an $\mathrm{AD}$ array, completely neglecting electron-electron interactions inside the 2DEG. At zero $B$, in the case of Coulomb interaction in a normal metal, this is a well-controlled approximation governed by the large parameters $k_{F} a_{B}$ and $k_{F} l_{S}$, where $a_{B}$ is the Bohr radius (and a characteristic screening length at zero $B$ ). At nonzero $B$, the situation appears to be much less trivial. Although in this paper we do not provide any treatment of the combined effect of the interactions and disorder on the CR line, a few comments are in order.

First, the Landau quantization enhances the role of the interaction. In particular, for weak $B$, the ground state of a disorder-free 2DEG is known ${ }^{25,26}$ to spontaneously break translational symmetry in a partially filled (i.e., highest occupied) Landau level. It is electrons from this level and those from the highest fully occupied level that are excited in the $\mathrm{CR}$ at zero temperature. On the other hand, the $\mathrm{CR}$ at zero wave vector in a one-component system of particles with a parabolic dispersion without external inhomogeneities is insensitive to the electron-electron interaction (Kohn's theorem ${ }^{27}$ ). Hence, it is only because of the combined effect of disorder and interactions that the latter can affect the CR line shape (e.g., Refs. 28 and 29 and references therein). At present, it is unclear what the resulting line shape of the dynamical response is for the system studied in Refs. 25 and 26 (see also Refs. 30-32) in the presence of disorder. How- 
ever, since electrons skipping around hard-wall AD's do not experience the Landau quantization (in this sense disorder is strong for them), we expect that their dynamical response has only weak interaction corrections (for $k_{F} a_{B} \gg 1$ and $\left.k_{F} R_{c} \gg 1\right)$. On the experimental side, the CR of strongly correlated particles has been investigated in the high- $B$ limit (e.g., Refs. 33-36 and references therein), but not for the translational-symmetry-broken state ${ }^{25,26}$ in weaker magnetic fields.

Second, because of electron-electron interactions, the quantity that is directly measured in far-infrared (e.g., Ref. 37) or microwave (e.g., Refs. 38-40) experiments may be related to the conductivity in a complex way. In the paper, we have provided explicit results for the conductivity: more specifically, for $S_{c}(\omega) \propto \operatorname{Re} \sigma_{x x}(\omega)+\operatorname{Im} \sigma_{x y}(\omega)$. We expect only small interaction corrections to $S_{c}(\omega)$. It is important, however, that the conductivity expresses the current as a response to the total (screened) electric field. As such, it is given by the irreducible, with respect to the Coulomb interaction, density-density response function $K\left(\mathbf{q}, \mathbf{q}^{\prime}, \omega\right)$. On the other hand, in contrast to dc measurements, what is probed directly in the ac transmission experiments is a response to the external field. The latter is given, in an operator form, by the reducible polarization $\epsilon^{-1} K$, where $\epsilon$ is the dielectric function. Put differently, the dissipated power is measured in units of the intensity of the incident wave. Since the measured absorption is increased near zeros of $\epsilon$, i.e., on resonance with plasma oscillations, the dynamical response of a 2DEG calculated in the paper is in general masked in the absorption experiments by the excitation of magnetoplasmons (for optical experiments with magnetoplasmons see Refs. 37,41-44,22, and 45-47). The measured quantity appears to depend in an essential way on the experimental setup (which may be very different; cf. Refs. 39,38, and 40).

Let us mention one more point related to edge magnetoplasmons in $\mathrm{AD}$ arrays, i.e., a soft mode of plasma oscillations localized near the sharp edges of AD's (Refs. 48 and 49; for experiments see Refs. 41-44,22, and 45-47). It is often asserted that there is an intimate connection between the collective edge excitations and skipping orbits. In fact, this notion may be very misleading. Indeed, the width of the strip around an $\mathrm{AD}$ in which the skipping orbits propagate along the edge is $2 R_{c}$. However, the current in the edge magnetoplasmon mode decays, in general, on a different scale: e.g., at $\omega \ll \omega_{c}$ and $R_{c} \gg a_{B}$ this scale is given ${ }^{49}$ by $R_{c}^{2} / a_{B} \gg R_{c}$. Hence, the main contribution to the edge magnetoplasmon current may come from electrons that do not at all collide with the AD. Since the frequency dependence of the dissipative response of the skipping orbits and that of the edge magnetoplasmons belong to different ranges of $\omega$, it appears to be quite possible that they can be measured separately in transmission experiments.

In conclusion, we have seen that the dynamical conductivity of electrons scattered on impenetrable AD's in the presence of a magnetic field reveals strong memory effects in the electron dynamics, associated with skipping orbits bound to AD's. These lead to the CR line shape which is not at all characterized by the Drude scattering rate. The contribution of the skipping orbits $S_{c}(\omega)$ is broadened on a scale of the cyclotron frequency $\omega_{c}$ and vanishes at $\omega_{c}$ in a nonanalytical way as $\left|\omega-\omega_{c}\right|$. Apart from these two features, $S_{c}(\omega)$ exhibits different behavior depending on the ratio of the cyclotron radius $R_{c}$ and the $\mathrm{AD}$ radius $a$. At large $R_{c} / a, S_{c}(\omega)$ oscillates with a period $\omega_{c}$ up to $\omega=\omega_{c} R_{c} / a$ and shows a series of square-root spikes for larger $\omega$. At small $R_{c} / a$, $S_{c}(\omega)$ has a hard gap between two sharp peaks located at $\omega \sim \omega_{c} R_{c} / a$ and $\omega=\omega_{c}$. We hope that these results will stimulate further experimental work on the ac conductivity in $\mathrm{AD}$ arrays.

\section{ACKNOWLEDGMENTS}

We thank L.W. Engel, R. Haug, F. Hohls, I.V. Kukushkin, U. Merkt, R.J. Nicholas, and J.H. Smet for discussions concerning the experiments. This work was supported by SFB 195 and the Schwerpunktprogramm "Quanten-HallSysteme" of the Deutsche Forschungsgemeinschaft, by INTAS Grants No. 99-1070 and 99-1705, and by RFBR Grants No. 99-02-17093 and 00-02-17002.
*Also at A.F. Ioffe Physico-Technical Institute, 194021 St. Petersburg, Russia.

${ }^{1}$ D. K. Ferry and S. M. Goodnick, Transport in Nanostructures (Cambridge University, Cambridge, United Kingdom, 1997).

${ }^{2}$ C. W. J. Beenakker and H. van Houten, in Semiconductor Heterostructures and Nanostructures [Solid State Phys. 44, 1 (1991)].

${ }^{3}$ D. Weiss, G. Lütjering, and K. Richter, Chaos, Solitons Fractals 8, 1337 (1997).

${ }^{4}$ R. Fleischmann, T. Geisel, R. Ketzmerick, and G. Petschel, Physica D 86, 171 (1995).

${ }^{5}$ G. M. Gusev, P. Basmaji, Z. D. Kvon, L. V. Litvin, Yu. V. Nastaushev, and A. I. Toropov, J. Phys.: Condens. Matter 6, 73 (1994).

${ }^{6}$ K. Tsukagoshi, S. Wakayama, K. Oto, S. Takaoka, K. Murase, and K. Gamo, Phys. Rev. B 52, 8344 (1995).

${ }^{7}$ G. Nachtwei, Z. H. Liu, G. Lütjering, R. R. Gerhardts, D. Weiss, K. von Klitzing, and K. Eberl, Phys. Rev. B 57, 9937 (1998).
${ }^{8}$ O. Yevtushenko, G. Lütjering, D. Weiss, and K. Richter, Phys. Rev. Lett. 84, 542 (2000).

${ }^{9}$ A. D. Mirlin, J. Wilke, F. Evers, D. G. Polyakov, and P. Wölfle, Phys. Rev. Lett. 83, 2801 (1999).

${ }^{10}$ A. D. Mirlin, D. G. Polyakov, F. Evers, and P. Wölfle, Phys. Rev. Lett. 87, 126805 (2001).

${ }^{11}$ D. G. Polyakov, F. Evers, A. D. Mirlin, and P. Wölfle, Phys. Rev. B 64, 205306 (2001).

${ }^{12}$ M. M. Fogler, A. Yu. Dobin, V. I. Perel, and B. I. Shklovskii, Phys. Rev. B 56, 6823 (1997).

${ }^{13}$ F. Evers, A. D. Mirlin, D. G. Polyakov, and P. Wölfle, Phys. Rev. B 60, 8951 (1999).

${ }^{14}$ É. M. Baskin, L. N. Magarill, and M. V. Éntin, Zh. Éksp. Teor. Fiz. 75, 723 (1978) [ Sov. Phys. JETP 48, 365 (1978)]; E. M. Baskin and M. V. Entin, Physica B 249-251, 805 (1998).

${ }^{15}$ A. V. Bobylev, F. A. Maaø, A. Hansen, and E. H. Hauge, Phys. Rev. Lett. 75, 197 (1995); J. Stat. Phys. 87, 1205 (1997). 
${ }^{16}$ A. Kuzmany and H. Spohn, Phys. Rev. E 57, 5544 (1998).

${ }^{17}$ M. M. Fogler and B. I. Shklovskii, Phys. Rev. Lett. 80, 4749 (1998).

${ }^{18}$ T. Ando, J. Phys. Soc. Jpn. 38, 989 (1975).

${ }^{19}$ D. Weiss, M. L. Roukes, A. Menschig, P. Grambow, K. von Klitzing, and G. Weimann, Phys. Rev. Lett. 66, 2790 (1991).

${ }^{20}$ R. Fleischmann, T. Geisel, and R. Ketzmerick, Phys. Rev. Lett. 68, 1367 (1992).

${ }^{21}$ J. Eroms, M. Zitzlsperger, D. Weiss, J. H. Smet, C. Albrecht, R. Fleischmann, M. Behet, J. de Boeck, and G. Borghs, Phys. Rev. B 59, R7829 (1999).

${ }^{22}$ E. Vasiliadou, R. Fleischmann, D. Weiss, D. Heitmann, K. von Klitzing, T. Geisel, R. Bergmann, H. Schweizer, and C. T. Foxon, Phys. Rev. B 52, R8658 (1995).

${ }^{23}$ It is worth mentioning that Eq. (13) describes the main features of the dynamic response of AD's with a smoother shape as well, provided their potential is sufficiently steep. Although the exact zeros will disappear, the oscillatory behavior of $S_{c}(\omega)$ is characteristic to the AD's as long as scattering on them has the form of skipping. The conditions of strong quasiclassical scattering off a well-defined boundary are satisfied reasonably well in most realizations of currently fabricated AD's.

${ }^{24}$ J. Wilke, A. D. Mirlin, D. G. Polyakov, F. Evers, and P. Wölfle, Phys. Rev. B 61, 13774 (2000).

${ }^{25}$ M. M. Fogler, A. A. Kulakov, and B. I. Shklovskii, Phys. Rev. B 54, 1853 (1996).

${ }^{26}$ R. Moessner and J. T. Chalker, Phys. Rev. B 54, 5006 (1996).

${ }^{27}$ W. Kohn, Phys. Rev. 123, 1242 (1961).

${ }^{28}$ H. Fukuyama, Y. Kuramoto, and P. M. Platzman, Phys. Rev. B 19, 4980 (1979).

${ }^{29}$ C. Kallin and B. I. Halperin, Phys. Rev. B 31, 3635 (1985).

${ }^{30}$ E. Fradkin and S. A. Kivelson, Phys. Rev. B 59, 8065 (1999).

${ }^{31}$ A. H. MacDonald and M. P. A. Fisher, Phys. Rev. B 61, 5724 (2000)

${ }^{32}$ F. von Oppen, B. I. Halperin, and A. Stern, Phys. Rev. Lett. 84, 2937 (2000).

${ }^{33}$ B. A. Wilson, S. J. Allen, Jr., and D. C. Tsui, Phys. Rev. B 24, 5887 (1981).
${ }^{34}$ M. J. Chou, D. C. Tsui, and G. Weimann, Phys. Rev. B 37, 848 (1988).

${ }^{35}$ M. Besson, F. Gornik, C. M. Engelhardt, and G. Weimann, Semicond. Sci. Technol. 7, 1274 (1992).

${ }^{36}$ G. M. Summers, R. J. Warburton, J. G. Michels, R. J. Nicholas, J. J. Harris, and C. T. Foxon, Phys. Rev. Lett. 70, 2150 (1993). Note that a weak dependence of the cyclotron frequency on spin orientation may become important for the explanation of the experimentally obtained line shape; see N. R. Cooper and J. T. Chalker, ibid. 72, 2057 (1994).

${ }^{37}$ E. Batke, D. Heitmann, and C. W. Tu, Phys. Rev. B 34, 6951 (1986).

${ }^{38}$ L. W. Engel, D. Shahar, C. Kurdak, and D. C. Tsui, Phys. Rev. Lett. 71, 2638 (1993).

${ }^{39}$ F. Hohls, U. Zeitler, and R. Haug, Phys. Rev. Lett. 86, 5124 (2001).

${ }^{40}$ P. D. Ye, L. W. Engel, D. C. Tsui, J. A. Simmons, J. R. Wendt, G. A. Vawter, and J. L. Reno, cond-mat/0103127 (unpublished).

${ }^{41}$ K. Kern, D. Heitmann, P. Grambow, Y. H. Zhang, and K. Ploog, Phys. Rev. Lett. 66, 1618 (1991).

${ }^{42}$ Y. Zhao, D. C. Tsui, M. Santos, M. Shayegan, R. A. Ghanbari, D. A. Antoniadis, and H. I. Smith, Appl. Phys. Lett. 60, 1510 (1992).

${ }^{43}$ A. Lorke, I. Jejina, and P. Kotthaus, Phys. Rev. B 46, 12845 (1992).

${ }^{44}$ K. Bollweg, T. Kurth, D. Heitmann, E. Vasiliadou, K. Eberl, and H. Brugger, Phys. Rev. B 52, 8379 (1995).

${ }^{45}$ K. Bollweg, T. Kurth, D. Heitmann, V. Gudmundsson, E. Vasiliadou, P. Grambow, and K. Eberl, Phys. Rev. Lett. 76, 2774 (1996).

${ }^{46}$ M. Hochgräfe, R. Krahne, Ch. Heyn, and D. Heitmann, Phys. Rev. B 60, 10680 (1999).

${ }^{47}$ S. Cinà, D. D. Arnone, H. P. Hughes, C. L. Foden, D. M. Whittaker, M. Pepper, and D. A. Ritchie, Phys. Rev. B 60, 7780 (1999).

${ }^{48}$ V. Fessatidis, H. L. Cui, and O. Kühn, Phys. Rev. B 47, 6598 (1993).

${ }^{49}$ S. A. Mikhailov and V. A. Volkov, Phys. Rev. B 52, 17260 (1995); S. A. Mikhailov, ibid. 54, 14293 (1996). 